\title{
Author Correction: Mechanical performance of ultra-high-performance strain-hardening cementitious composites according to binder composition and curing conditions
}

\author{
Min-Jae Kim ${ }^{1} \cdot$ Taekgeun $\mathrm{Oh}^{1} \cdot$ Doo-Yeol Yoo ${ }^{1}$ (D)
}

Published online: 21 February 2022

c) Wroclaw University of Science and Technology 2022

\section{Correction to: \\ Archives of Civil and Mechanical Engineering (2022) 22:63 https://doi.org/10.1007/s43452-022-00385-w}

In this article the statement in the Funding information section was incorrectly given as 'This research was supported by a grant (21SCIP-B146646-04) from the Construction Technology Research Project funded by the Ministry of Land, Infrastructure, and Transport of the Korean government.' and should have read
'This work was supported by the National Research Foundation of Korea (NRF) grant funded by the Korea government (MSIT) (No. 2021R1A2C4001503)'.

The original article can be found online at https://doi.org/10.1007/ s43452-022-00385-w.

Doo-Yeol Yoo

dyyoo@hanyang.ac.kr

Min-Jae Kim

mandufa345@ hanyang.ac.kr

Taekgeun Oh

theia8632@hanyang.ac.kr

1 Department of Architectural Engineering, Hanyang

University, 222 Wangsimni-ro, Seongdong-gu, Seoul 04763,

Republic of Korea 